\title{
Some clinical and pathologic observations on chronic myelopathy: a variant of multiple sclerosis
}

\author{
B G Weinshenker, J J Gilbert, G C Ebers
}

\begin{abstract}
Four patients with progressive demyelinating myelopathy with symptoms spanning six to 25 years are described. There was no clinical evidence of dissemination of lesions in the central nervous system. Radiological evidence of dissemination was present in two cases; in one this was absent at the time of presentation and was only demonstrated after six years of progressive unifocal disease. In one case, pathological examination revealed a solitary area of chronic demyelination. In all cases but one, oligoclonal bands were detected on cerebrospinal fluid (CSF) electrophoresis. Some cases of chronically progressive myelopathy result from focal demyelination in the absence of a second lesion demonstrable by clinical, radiographic or necropsy examination.
\end{abstract}

Traditionally, the diagnosis of clinically definite multiple sclerosis has required demonstration of at least two white matter lesions disseminated in both space and time. Paraclinical, radiological and electrophysiological tests frequently demonstrate additional lesions when only one can be determined clinically. ${ }^{12}$ A frequent setting for diagnostic confusion is the occurrence of monosymptomatic progressive spastic myelopathy. After diagnoses such as $B_{12}$ deficiency, motor neuron disease and structural myelopathies have been excluded, many cases remain diagnostic enigmas. A large percentage of these are suspected to have multiple sclerosis, based on the results of cerebrospinal fluid (CSF) electrophoresis, electrophysiological testing ${ }^{3}$ and recently on magnetic resonance imaging (MRI). ${ }^{4}$ In cases without these paraclinical abnormalities the presumption has been that these patients do not have multiple sclerosis or that they will develop further clinical abnormalities which will clarify the diagnosis.

To our knowledge, progressive myelopathy due to a solitary demyelinating lesion has yet to be documented pathologically. We present four patients with clinically isolated progressive myelopathy, with a spectrum of radiological and pathological findings. All but one had an oligoclonal band pattern on CSF electrophoresis suggesting that demyelination was the underlying pathophysiology. In one case we were able to document pathologically that there was a single isolated plaque of demyelination in the spinal cord despite a history of progressive myelopathy of over 25 years duration.

\section{Case reports}

Patient 1: Clinically isolated myelopathy with radiological and pathological dissemination The patient was a 73 year old female with progressive lower extremity paraesthesiae and weakness of 20 years duration. The onset was subacute and associated with low back pain. She had residual leg weakness and numbness. After negative myelography, she was given a diagnosis of multiple sclerosis. The patient continued to develop progressive lower extremity weakness and in June 1984 developed a sudden worsening of weakness and numbness which was not associated with back pain or other clear precipitating factors. Her symptoms resolved after rest in hospital and she returned to her baseline level of functioning, being able to walk with a cane.

At that time neurological examination revealed normal fundi and only slight limitation of upward gaze. Strength, tone, bulk and coordination were normal in the upper extremities. The lower extremities were both weak, with greater weakness on the right side than on the left. She could barely lift the extended left leg off the bed. Both plantar responses were upgoing. There was a marked decrease in vibration sense below the iliac crest and some loss of proprioception at the toes bilaterally. There was diminished pinprick sensitivity below $L 2$ on the right and T11 on the left. Auditory evoked responses were normal. A myelogram was normal. CSF protein was normal and oligoclonal banding was absent.

She was readmitted for investigation of a generalised seizure in January 1986, for which no cause was established. CT revealed mild cerebral atrophy. MRI $(0 \cdot 15$ Tesla, Technicare Corporation Solon, Ohio) of the head and cervical spine revealed extensive periventricular increase in signal on $\mathrm{T} 2$-weighted images with small foci of increased signal in the left parietal white matter and right frontal region; no focal abnormalities were demonstrated within the cord. The patient died two months later from a myocardial infarct. Necropsy revealed multiple grey firm lesions in the white matter of the cerebral hemispheres, primarily but not exclusively periventricular in distribution. Microscopically, there was focal demyelination and perivascular cuffing in the cerebral hemispheres, pons and cervical cord. These findings were typical of multiple sclerosis.

Patient 2: Clinically and radiologically isolated myelopathy

The patient is a 56 year old female with progressive weakness of her legs, worse on the 
left, over a period of 10 years. Her symptoms began six weeks after immunisation for influenza. She complained of tingling in her left leg which resolved after one year although she continued to experience a persistent heavy feeling in that leg. Subsequently, she developed progressive weakness of both legs. She had no symptoms referrable to cranial nerves or mental function. The patient had intermittent bilateral numbness associated with blanching of her fingertips and had been diagnosed as having Raynaud's syndrome. Sphincter function was normal.

Examination revealed no abnormality of cranial nerves. Tone was increased in the left arm and leg. There was no weakness of the left hand, although rapid alternating movements were impaired with that hand. There was bilateral weakness (MRI grade 4) of the lower extremities although this was worse on the left side than on the right. The tendon reflexes were pathologically brisk on the left side with a left Babinski sign. MRI of the head (0.15 Tesla, Technicare) showed no abnormality. CSF studies showed a normal IgG/albumin ratio and oligoclonal banding on agarose electrophoresis.

Patient 3: Clinically and radiologically isolated myelopathy but prolonged follow up

demonstrated MRI dissemination

The patient is a 56 year old male who at the age of 50 developed insidiously progressive numbness and coldness of his left arm over several months. He complained of soreness in his left arm. His condition progressed slowly and he came to neurological attention when he developed weakness in his left leg four years later. Myelography suggested the presence of a compressive cervical disc at C5-6 and led to discectomy and fusion at that level. His condition continued to progress post-operatively with increasing weakness of his left arm and leg. He complained of Lhermitte's symptom in the left arm and leg with neck flexion and developed minor difficulties with bladder emptying.

Examination revealed no abnormalities of mental status or cranial nerves. There was weakness and wasting of muscles of the left shoulder girdle, arm and forearm with particularly severe weakness of finger extensors and interossei of the left hand. There were no fasciculations. Deep tendon reflexes were pathologically brisk in all four limbs, especially in the left arm and leg. Sensory examination revealed no abnormality. There were no cerebellar signs. Visual evoked responses revealed no abnormality. Brain stem auditory evoked responses revealed an abnormality in the right pons, in that wave $\mathrm{V}$ was seen inconsistently and appeared delayed. Spinal fluid analysis revealed a normal IgG/albumin ratio but oligoclonal banding was detected on CSF electrophoresis. MRI (0.15 Tesla, Technicare) revealed focal atrophy and increased signal in the centre of the cord at the C2-3 level; MRI of the head revealed no abnormality. Serial follow up revealed continued progression of the leftsided weakness, but no development of new symptoms. MRI (1.5 Tesla, General Electric, Milwaukee, Wisconsin) six years after the onset of his symptoms revealed multiple areas of high intensity signal in the subcortical and periventricular regions which were compatible with multiple sclerosis.

\section{Patient 4: Clinically and pathologically isolated myelopathy}

The patient was a 59 year old male who experienced the onset of neurological symptoms approximately 25 years before his death in 1979. He experienced progressive weakness of both legs, the left leg being consistently weaker than the right. He also had a disturbance of
Figure 1 Focal acute demyelination of crossing fibres in basis pontis. $(\times 24)$. Solochrome R/Neutral Red.

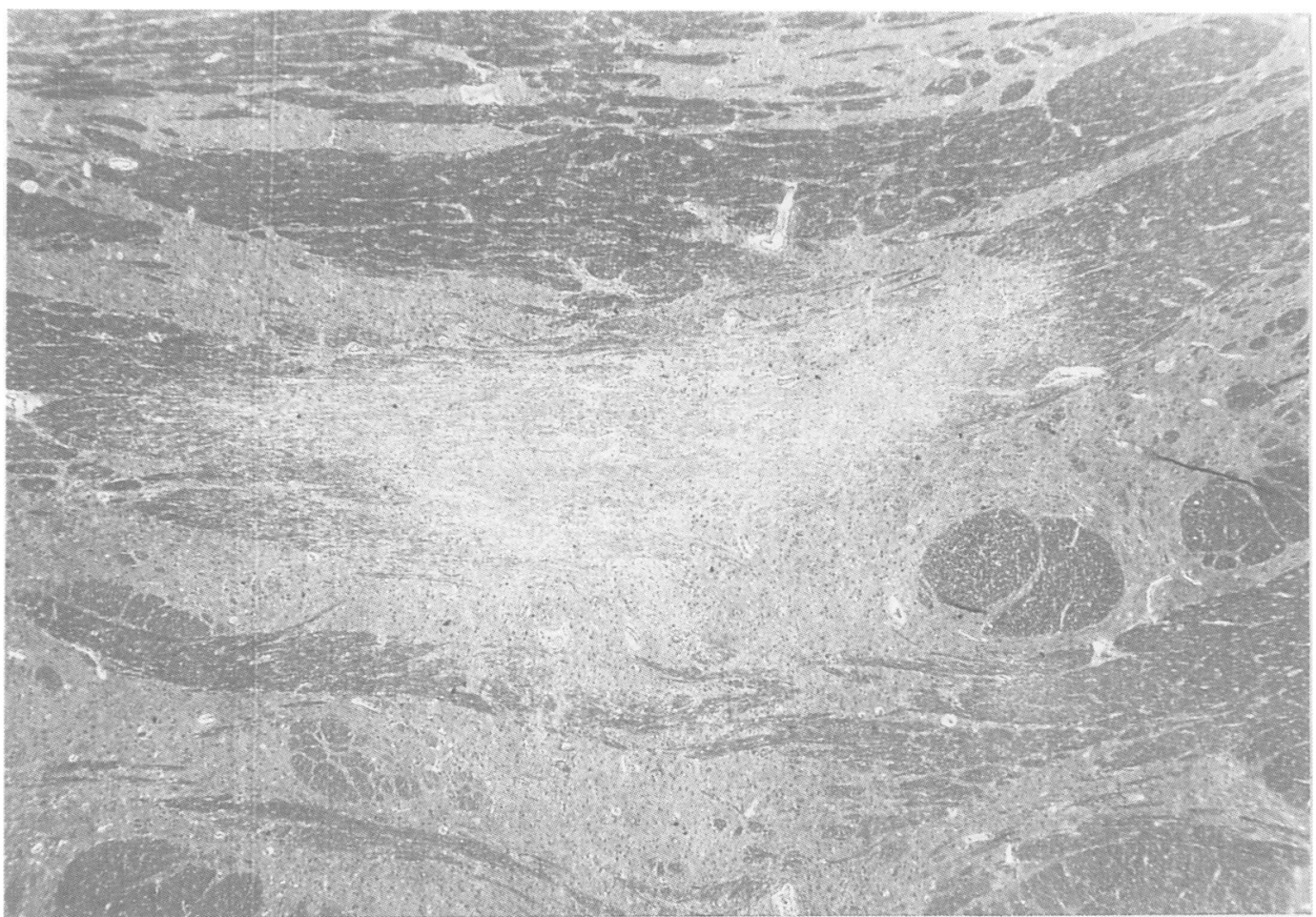


Figure 2 Mid thoracic spinal cord demyelinating plaque predominantly involving the dorsal columns. Focal areas of demyelination are also apparent in the lateral columns. Solorchrome R/Neutral Red.

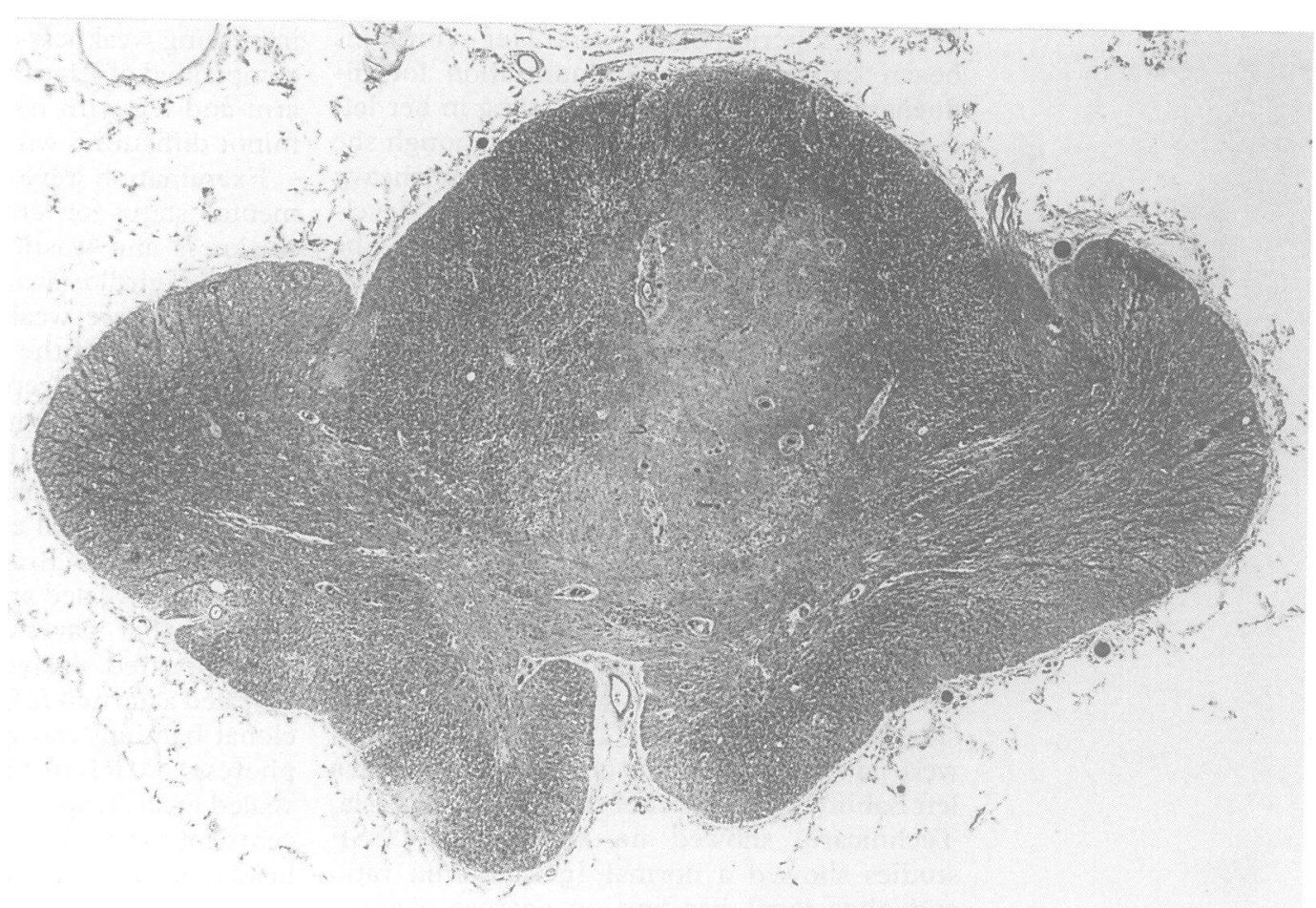

bladder function. There were no alterations in cognitive function, vision, speech or upper extremity function. His disease progressed slowly and he was able to walk with a cane until two years before his death.

Following the death of his wife in 1977, he began to drink excessively and experienced a further decline of neurological function in his legs which led to his confinement to a wheelchair. He then developed decubitus ulcers. In 1977 he was examined and found to have horizontal gaze-evoked nystagmus on looking to either side but no other cranial nerve abnormalities. There was marked spastic weakness of the left leg and mild weakness of the right. Proprioception was diminished in both legs. The patient was admitted to hospital in 1979 with a two week history of diarrhoea and flulike illness. He had a pulmonary infiltrate and was suspected of having pneumonia. Ophthalmological examination revealed no abnormality. He developed acute abdominal pain in hospital and signs of peritonitis. Hours before his demise his serum sodium fell to $118 \mathrm{mmol} / 1$ and he developed prerenal azotaemia. He died despite vigorous fluid resuscitation.

The general necropsy revealed diffuse wasting, flexion contractures and decubitus ulcers. There was generalised peritonitis due to a ruptured bladder diverticulum. Sectioning of the brain showed no significant atrophy or abnormality of the hemispheres. Microscopic analysis of numerous sections of periventricular white matter as well as cerebellar cortex failed to reveal evidence of demyelination. In the rostral basis pontis, an area of acute bland demyelination without inflammation and without significant axonal loss was seen. This focal lesion was compatible with central pontine myelinolysis (fig 1). Close sectioning of the spinal cord revealed an area of discolouration of the mid-thoracic white matter which was 1.5 $\mathrm{cm}$ in length. This lesion, under the microscope, consisted of patchy asymmetric destruction of white matter with relative preservation of grey matter (fig 2). There was marked gliosis, astrocytosis and Schwannosis especially in the dorsal root entry zones. There was evidence of tract degeneration, the corticospinal tract in the lower thoracic and lumbosacral cord and the spinocerebellar and fasciculus gracillis in the cervical cord. The appearance of the cord was consistent with a solitary old demyelinating plaque. Electrophoresis of CSF obtained at the time of necropsy demonstrated an oligoclonal band pattern.

\section{Discussion}

All four cases described had a long standing chronically progressive myelopathy with no clinical evidence of involvement of the nervous system at other sites. Radiological evidence of dissemination of lesions in the central nervous system by MRI was present in two patients. Oligoclonal band pattern was demonstrated by agarose electrophoresis of CSF in three of the four cases. In no case was an alternative diagnosis other than multiple sclerosis demonstrable.

Patient 1 demonstrates the commonest situation; laboratory support enables a diagnosis of multiple sclerosis to be reached antemortem when a definite clinical diagnosis of multiple sclerosis would have been otherwise impossible. Patient 4 represents the other extreme of the spectrum of patients with isolated chronically progressive myelopathy. There was pathological evidence of a solitary demyelinating lesion at a site appropriate to the clinical picture. The area of bland basis pontis demyelination was recent by histological criteria, and had the appearance of central pontine 
myelinolysis, which is the result of a metabolic result. ${ }^{6}$

In the case of patient 3 , an area of focally increased signal on T2-weighted MRI with associated atrophy in an area appropriate to the clinical lesion was discovered. No radiological evidence of dissemination of lesions was present until six years after the onset of progressive myelopathy. In patient 2 , no lesion was present on MRI of the head, and, by clinical criteria, the progressive course could be accounted for entirely by a single focal lesion.

One must be cautious in the interpretation of negative MRI results. While it is the most sensitive of investigations for the demonstration of lesions disseminated in space, current estimates of its sensitivity in the diagnosis of suspected multiple sclerosis are $95 \%{ }^{7}$ Technical considerations including field strength of the magnet, spatial resolution and the scanning protocol could potentially affect sensitivity of the test. However, in patients with chronically progressive disease that has been present for many years, the sensitivity approaches $100 \%$. Furthermore, specificity of multifocal white matter abnormalities in the elderly is imperfect; quite frequently, these abnormalities are associated with hypertension and are suspected to be vascular lesions. ${ }^{8}$ Specificity of isolated MRI abnormalities in the spinal cord is occasionally uncertain. However, prolonged observation and serial MRI in the third patient failed to reveal evidence of syrinx or tumour, and the additional presence of oligoclonal banding likely improved the predictive value in this case.

Paty ${ }^{3}$ has reviewed the literature and studied a series of 72 patients with chronically progressive myelopathy without clinical evidence of other lesions; he concludes that the percentage of such patients having multiple sclerosis may be as high as $44 \%$ when ancillary electro- physiological and radiological tests, as well as spinal fluid electrophoresis are considered. Miska $^{4}$ in a similar analysis of 20 patients found that up to $85 \%$ of patients with chronic myelopathy of unknown aetiology may have multiple sclerosis when MRI is added to the diagnostic armamentarium. Miller ${ }^{5}$ found abnormalities on MRI of the brain in $82 \%$ of 89 patients with chronic nonprogressive spinal cord syndromes, and an intrinsic cord abnormality in $62 \%$ of 58 patients with a chronic cervical cord syndrome. We suggest that others with negative ancillary studies might also have demyelinating disease as the cause of their myelopathy despite normal MRI findings. This study establishes that a solitary demyelinating lesion can produce chronic myelopathy without pathological evidence of dissemination of lesions. The association with oligoclonal bands and lack of another explanation for the clinical findings suggests that these cases are variants of multiple sclerosis.

1 Poser CM, Paty DW, Scheinberg L, et al. New diagnostic criteria for multiple sclerosis: guidelines for research protocols. Ann Neurol 1983;13:227-31.

2 Poser CM, Paty DW, McDonald WI, Scheinberg L, Ebers GC, eds. The Diagnosis of Multiple Sclerosis. New York: Thieme-Stratton, 1984:103-204.

3 Paty DW, Blume WT, Brown WF, Jautoul N, Kertesz A, McInnis W. Chronic progressive myelopathy: investigation with CSF electrophoresis, evoked potentials, and CT scan. Ann Neurol 1979;6:419-24.

4 Miska RM, Pojunas KW, McQuillen MP. Cranial magnetic resonance imaging in the evaluation of myelopathy of undetermined etiology. Neurology 1987;37:840-3.

5 Miller DH, McDonald WI, Blumhurdt LD, et al. Magnetic resonance imaging in isolated noncompressive spinal cord resonance imaging in isolated noncompressiv
syndromes. Ann Neurol 1987;22:714-23.

6 Sterns RH, Riggs JE, Schochet SS, Jr. Osmotic demyelination following correction of hyponatremia. $N$ Eng $J$ Med 1986;314:1535-42.

7 Paty DW, Hashimoto SA, Hooge JP, et al. MRI in the diagnosis of multiple sclerosis: a prospective study with comparison of clinical evaluation, evoked potentials, oligoclonal banding, and CT. Neurology 1988;38: 180-5. 8 Hachinski VC, Potter P, Merskey H. Leuko-Araiosis. Arch
Neurol 1987;44:21-3. 